\title{
SOME REMARKS ON ABSOLUTE MATHEMATICS
}

\section{Stanislaw Betley}

\begin{abstract}
We calculate Hochschild cohomology groups of the integers treated as an algebra over so-called "field with one element". We compare our results with calculation of the topological Hochschild cohomology groups of the integers - this is the case when one considers integers as an algebra over the sphere spectrum.
\end{abstract}

\section{INTRODUCTION.}

Absolute mathematics means here computations over field $F_{1}$ with one element in the sense of Soule and others (see $[\mathrm{S}]$ or $[\mathrm{KOW}]$ ). There are two reasons for writing this short note. First of all we want to pursue a little further calculations of Hochschild cohomology of $Z$ treated here as $F_{1}$-algebra, started in [KOW]. This theory is highly nontrivial and interpretation of nontrivial classes is still to be discovered. But the more important reason is contained in Section 4. The idea of treating $Z$ as an algebra over some ring in a nontrivial way has been stimulating for topologists for many years. The topological approach to this problem can be sketched in a few words. One identifies $Z$ with Eilenberg-MacLane spectrum $H(Z)$ and then considers it as an algebra spectrum over sphere spectrum $S$. This lead to the definition of topological Hochschild homology of $Z$ which is highly nontrivial, contrary to the ordinary Hochschild theory over $Z$. So for $Z$ we have two nontrivial Hochschild type (co)homology theories: topological Hochschild (co)homology, which should be considered as ordinary Hochschild theory over $S$ and Hochschild (co)homology over $F_{1}$. In Section 4 we describe how these two theories are related. From this we derive vanishing results on Hochschild cohomology over $F_{1}$ for certain rings of matrices which seem to be of independent interest (see for example $[\mathrm{KOW}]$ ).

\section{HOCHSCHILD COHOMOLOGY OF Z OVER $F_{1}$.}

In our treatment of absolute mathematics we will follow [KOW]. Recall, that viewing a ring $R$ as $F_{1}$-algebra means that we forget the additive structure of $R$ so we restrict ourselves to the multiplicative monoid structure of $R$. To avoid misunderstandings we will use constantly bold notation for monoids and normal one for other algebraic objects like rings, abelian groups, bimodules, etc. Hence, when we use notation $\mathbf{R}$ for the ring $R$, it means that we treat $R$ only as the multiplicative monoid.

For example, the absolute derivations of $R$ with values in an $R$-bimodule $M$ (notation $\operatorname{Der}_{F_{1}}(R, M)$ ) are maps $D: R \rightarrow M$ (not necessary linear) which satisfy the Leibniz rule

$$
D(a b)=D(a) b+a D(b)
$$

As it was shown in $[\mathrm{KOW}$, Theorem 1] there is a direct product decomposition

$$
\operatorname{Der}_{F_{1}}(\mathbf{Z}, Z)=\prod_{p: \text { prime }} Z f_{p}
$$


where $f_{p}: \mathbf{Z} \rightarrow Z$ is defined by the formula $f_{p}(x)=\frac{x}{p} \cdot \operatorname{ord}_{p}(x)$. The number $\operatorname{ord}_{p}(x)$ means here $p$-order of $x$ and it is easy to check that $f_{p}$ satisfies Leibniz rule.

Let $R$ be a ring and $M$ an $R$-bimodule. Define (see [KOW, section 1.6]) the cochain complex $\left(C^{n}(R, M), \delta^{n}\right)$ where the nth group is given by the formula

$$
C^{n}(R, M)=\operatorname{Map}\left(R^{n}, M\right)
$$

and

$$
\begin{aligned}
\left(\delta^{n} f\right)\left(a_{1}, \ldots, a_{n+1}\right)= & a_{1} f\left(a_{2}, \ldots, a_{n+1}\right)+\sum_{i=1}^{n}\left(-1^{i}\right) f\left(a_{1}, \ldots, a_{i} a_{i+1}, \ldots, a_{n+1}\right) \\
& +\left(-1^{n+1}\right) f\left(a_{1}, \ldots, a_{n}\right) a_{n+1}
\end{aligned}
$$

The cohomology of this complex will be called the absolute Hochschild cohomology of $R$ with coefficients in $M$ and denoted $H_{F_{1}}^{*}(\mathbf{R}, M)$. It is easy to check that Theorem 1 of [KOW] calculates $H_{F_{1}}^{1}(\mathbf{Z}, Z)=\operatorname{Der}_{F_{1}}(\mathbf{Z}, Z)$.

One easily identifies

$$
M a p\left(R^{n}, M\right)=\operatorname{Hom}_{Z}\left(Z\left[R^{n}\right], M\right)=H_{\mathrm{om}}\left(Z[R]^{\otimes n}, M\right)
$$

In other words our absolute Hochschild cohomology theory can be identified with the Hochschild cohomology groups over $Z$ of the monoid ring $Z[\mathbf{R}]$ with coefficients in $M$. This means that we can extend the definition of $H_{F_{1}}^{*}$-groups to all monoids with coefficients in arbitrary bimodules over their monoid rings. In this section we concentrate on multiplicative monoids of integers $(\mathbf{Z})$ and natural numbers $(\mathbf{N})$ and their zero-free versions $\mathbf{Z}^{*}$ and $\mathbf{N}^{*}$.

2.1.Theorem: The embedding $\mathbf{N}^{*} \rightarrow \mathbf{N}$ induces an isomorphism on $H_{F_{1}}^{*}$-groups with $Z$ coefficients, where $\mathbf{N}$ acts on $Z$ by multiplication. The same is true for the embedding $\mathbf{Z}^{*} \rightarrow \mathbf{Z}$.

Proof. Choose a natural number $n$. We show first that every element of $H_{F_{1}}^{n}(\mathbf{N}, Z)$ can be represented by a cocycle which vanishes on all $n$-tuples which contain a zero element.

Let $f \in C^{n}(\mathbf{N}, Z)$ be a cocycle. Assume first that $f(0, \ldots, 0)=a \neq 0$. We will find a representative of the same cohomology class which vanishes on $(0, \ldots, 0)$. The element $f$ is a cocycle so its coboundary is 0 . When we calculate $\delta^{n} f(0, \ldots, 0)$ we obtain $-a$ or 0 depending on the parity of $n$. In the first case we do nothing because it means that $a$ has to be zero anyway. In the second situation we replace $f$ by $f-\delta^{n-1} g$ where $g \in C^{n-1}(\mathbf{N}, Z)$ is defined by the following rule: $g(0, \ldots, 0)=a$ and $g$ is 0 at any other point.

Now we will continue our proof by induction. Assume that the cocycle $f \in C^{n}(\mathbf{N}, Z)$ vanishes at all points $\left(x_{1}, \ldots, x_{n}\right)$ which contain at least $k+1$ zeros and $f\left(y_{1}, \ldots, y_{n}\right)=$ $b \neq 0$ where the tuple $Y=\left(y_{1}, \ldots, y_{n}\right)$ has $k$ zeros. But to proceed further we need some more notation. Any maximal subsequence of $Y$ of length $s$ of the type $\left(y_{i}, y_{i+1}, \ldots, y_{i+s}\right)$ consisting of zeros only will be called a s-period of $Y$. Write $Y\left(e_{1}, \ldots, e_{i}, o_{1}, \ldots, o_{j}\right)$ for a 
$n$-tuple which has $i$ even periods of length $e_{s}, s=1, \ldots, i$ and $j$ odd ones of corresponding length $o_{t}, t=1, \ldots, j$. In such situation we will say that $Y$ is of the type $(i, j)$.

Assume first that $Y$ is of type $(0, j)$. Then

$$
0=\delta^{n} f\left(Y\left(o_{1}+1, o_{2}, \ldots, o_{j}\right)\right)= \pm f(Y)= \pm b
$$

by the assumption on $f$, so $b$ has to be 0 . Now assume that $Y$ is of type $(i, 0)$. Define $g \in C^{n-1}(\mathbf{N}, Z)$ by the formula $g\left(Y\left(e_{1}-1, e_{2}, \ldots, e_{i}\right)\right)= \pm b$ and $g$ is 0 at any other point. Whether we should have here plus or minus depends on the parity of the first term of the index in the period corresponding to $e_{1}$. Then

$$
\left(f-\delta^{n-1} g\right)(Y)=0
$$

and

$2.1 .1 \delta^{n-1} g$ is 0 everywhere but $Y$.

Hence the set of points with $k$ zeros for which $f-\delta^{n-1} g$ is non zero is smaller than such a set for $f$. So subtracting $\delta^{n-1} g$ like above for all points $Y$ of type $(., 0)$ we can make our cocycle vanish at all points with $k$ zeros and only even periods (we are in a product situation so infinite sums are allowed).

The situation, with even and odd periods is a little more complicated to describe. Of course it is very easy to make $f$ vanish at a particular $Y$ by subtracting some boundary element as previously. But we have to be sure that we will not increase the set of points with $k$ zeros at which our cocycle is non-zero. Analyze first the case when $Y$ is of type $(i, 1)$. Put $g \in C^{n-1}(\mathbf{N}, Z)$ to be defined by the formula

$$
\text { for any } t \quad g\left(Y\left(e_{1}, \ldots, e_{t}-1, \ldots, e_{i}, o_{1}\right)\right)= \pm f\left(Y\left(\ldots, e_{t}-1, \ldots, o_{1}+1\right)\right)
$$

and is 0 otherwise, where the choice of sign depends on the parity of the first term of the index in the period corresponding to $e_{t}$. We have to show that

2.1.2. $f-\delta^{n-1} g$ vanishes at $Y$.

Let us start from the calculation of $\delta^{n} f\left(Y\left(\ldots, o_{1}+1\right)\right)$. Because $f$ is a cocycle and vanishes at all points which contain at least $k+1$ zeros we get:

$$
0=\delta^{n} f\left(Y\left(\ldots, o_{1}+1\right)\right)=f(Y)+\sum_{t=1}^{i} f\left(Y\left(\ldots, e_{t}-1, \ldots, o_{1}+1\right)\right)
$$

where on the right hand side, in order to simplify notation, we did not put signs preceding all terms. These signs depend on the parity of the indices of the first terms of periods (as previously). Hence up to signs we can say that

$$
f(Y)=\sum_{t=1}^{i} f\left(Y\left(\ldots, e_{t}-1, \ldots, o_{1}+1\right)\right)
$$

On the other hand (up to signs as above) we calculate that 


$$
\delta^{n-1} g(Y)=\sum_{t=1}^{i} f\left(Y\left(\ldots, e_{t}-1, \ldots, o_{1}+1\right)\right)
$$

by the definition of $g$. Hence 2.1.2 is proven.

Moreover observe that $\delta^{n-1} g$ can be nonzero at a point with $k$ zeros only if this tuple equals $Y\left(\ldots, e_{t}-1, \ldots, o_{1}+1\right)$ for some $t \in\{1, \ldots, i\}$. But at such tuples $f$ and $g$ agree by the definition of $g$. Hence again the set of points with $k$ zeros for which $f-\delta^{n-1} g$ is non zero is smaller than such a set for $f$.

Now we have to treat the general case of $Y$ of the type $(i, j)$. We will write formulas only for $i \leq j$. The opposite situation should be done the same way and is left to the reader as an exercise. For any $s=1, \ldots, i$ let $Y_{s}$ denote the point in which $s$-even periods where shortened by 1 and $s$-1-odd periods where enlarged by 1 excluding the first odd period from extending procedure. Correspondingly, let $Y_{1, s}$ be the same as $Y_{s}$ with the first odd period extended also by 1 . Define $g$ by the formula: for any $s=1, \ldots, i$ and any choice of periods we have

$$
g\left(Y_{s}\right)=f\left(Y_{1, s}\right)
$$

and zero otherwise. Then the analogous calculations as above give us that $f-\delta^{n-1} g$ vanishes at $Y$ and the set of points with $k$ zeros for which $f-\delta^{n-1} g$ is non zero is smaller than such a set for $f$, as previously. The idea behind this construction is simple: when we increase the number of odd periods we increase the set of points at which $\delta^{n-1} g$ can be nontrivial (these are the points $Y_{1, s}$ ) so we have to make sure that $\delta^{n-1} g$ agrees at these points with $f$.

Now we can finish the proof of 2.1. Observe that any $f \in C^{n}(\mathbf{N}, Z)$ can be uniquely written $f=f_{1}+f_{0}$ where $f_{1}$ vanishes on all tuples with some coordinates equal to 0 and $f_{0}$ vanishes on all tuples without zeros. This decomposition is preserved by the boundary operators and gives us a decomposition of our cochain complex as a sum $C^{*}(\mathbf{N}, Z)=$ $C_{1}^{*} \oplus C_{0}^{*}$. Our considerations above show that $C_{0}^{*}$ has trivial cohomology. So we have our theorem for $\mathbf{N}$. It is obvious that the monoid $\mathbf{Z}$ can be treated similarly.

2.2.Remark: Observe that theorem 2.1 is valid for any commutative monoid $\mathbf{M}$ without 0 divisors with coefficients taken in any abelian group on which $\mathbf{M}^{*}$ acts by monomorphisms and $0 \in \mathbf{M}$ acts as multiplication by 0 .

Let $C_{2}$ denote the cyclic group of two elements. Identifying $C_{2}$ with $\{-1,1\}$ we see that $\mathbf{Z}^{*}=\mathbf{N}^{*} \times C_{2}$. We have Kunneth-type spectral sequence for the product of monoids and $C_{2}$ is a group so cohomology groups of its monoid ring are the same as cohomology of the group with conjugate action on coefficients. It means that in our case relevant are cohomology of the group $C_{2}$ with coefficients in the trivial module $Z$. These groups are given in every textbook containing the notion of group (co)homology. So everything that was done so far can be summarized in the statement that absolute cohomology $H_{F_{1}}^{*}(\mathbf{Z}, Z)$ will be fully understood if we know $H_{F_{1}}^{*}\left(\mathbf{N}^{*}, Z\right)$. 
Observe, that Theorem 1 of $[\mathrm{KOW}]$ that was quoted above gives us the calculation of $H_{F_{1}}^{1}\left(\mathbf{N}^{*}, Z\right)=H_{F_{1}}^{1}(\mathbf{Z}, Z)$ and the elements $f_{p}$ are well defined in $C^{1}\left(\mathbf{N}^{*}, Z\right)$.

2.3.Proposition: For any cocycle $f \in C^{n}\left(\mathbf{N}^{*}, Z\right)$ we can find a cocycle

$$
f^{\prime} \in C^{n}\left(\mathbf{N}^{*}, Z\right)
$$

which represents the same cohomology class as $f$ and which vanishes on all tuples which contain 1.

We will give only a proof of this proposition in two particular cases, because we do not have so far any application of this proposition in full generality. But the careful reader should notice immediately, that an analysis from the proof of Theorem 2.1 can be done with 0 replaced by 1 .

Partial Proof. Assume first that our cocycle $f$ is non trivial at $(1, \ldots, 1)$. Then by calculating $\delta^{n}(1, \ldots, 1)$ we get that $n$ has to be odd. But then $f=\delta^{n-1} g$, where $g$ is the $(n-1)$ cochain which at $(1, \ldots, 1)$ is equal to $f(1, \ldots, 1)$ and is zero otherwise.

Secondly assume that $f(1, \ldots, 1)=0$ and $f(a, 1, \ldots, 1)=b \neq 0$. Calculate $\delta^{n} f(a, 1, \ldots, 1)$. One gets immediately that $n$ has to be odd. Then $f=\delta^{n-1} g$, where $g$ is the $(n-1)$ cochain which at $(a, 1, \ldots, 1)$ is equal to $-f(a, \ldots, 1)$ and is zero otherwise. Case $(1, \ldots, 1, a)$ can be treated similarly.

Of course, it is easy to check by a direct computation that for any $n$ and any sequence of prime numbers $\left(p_{1}, \ldots, p_{n}\right)$ we have well defined cocycles in $H_{F_{1}}^{n}\left(\mathbf{N}^{*}, Z\right)$ given by the formulas

$$
f\left(a_{1}, \ldots, a_{n}\right)=f_{p_{1}}\left(a_{1}\right) \cdot \ldots \cdot f_{p_{n}\left(a_{n}\right)}
$$

For the increasing sequence $\left(p_{1}<p_{2}<, \ldots,<p_{n}\right)$ we will denote them later as $f_{\left(p_{1}, \ldots, p_{n}\right)}$.

In section 3 we will show in full generality that $H_{F_{1}}^{n}\left(\mathbf{N}^{*}, Z\right)$ is equal to the direct product of $Z$ indexed by all $f_{\left(p_{1}, \ldots, p_{n}\right)}$ defined above. Below we give a direct calculation of this fact in the case $n=2$.

2.4. Theorem: $H_{F_{1}}^{2}\left(\mathbf{N}^{*}, Z\right)$ is equal to the direct product of $Z$ indexed by all pairs of distinct prime numbers $(p, q)$ with $p<q$.

Proof: Observe, that for any pair of distinct prime numbers $p$ and $q$ we have the element $f_{p, q} \in C^{2}\left(\mathbf{N}^{*}, Z\right)$ defined by the formula

$$
f_{p, q}(a, b)=f_{p}(a) f_{q}(b)
$$

It is easy to check that for any $p, q$ this is a cocycle:

$$
\begin{gathered}
\delta^{2} f_{p, q}(a, b, c)=a f_{p}(b) f_{q}(c)-f_{p}(a b) f_{q}(c)+f_{p}(a) f_{q}(b c)-f_{p}(a) f_{q}(b) c \\
=a f_{p}(b) f_{q}(c)-a f_{p}(b) f_{q}(c)-b f_{p}(a) f_{q}(c)+b f_{p}(a) f_{q}(c)+c f_{p}(a) f_{q}(b)-f_{p}(a) f_{q}(b) c=0
\end{gathered}
$$


by Leibniz rule.

Moreover, for any $f \in C^{1}\left(\mathbf{N}^{*}, Z\right)$ we have the formula

$$
\delta^{1}(f)(a, b)=a f(b)-f(a b)+b f(a)
$$

which is invariant under permutation $a \leftrightarrow b$ so any coboundary satisfies this symmetry. On the other hand any product of elements of the type $f_{p, q}$ with $p<q$ is not symmetric in this sense. Hence we know that the product of $Z$ indexed by the set $\{(p, q) ; p, q$ primes $p<q\}$ is contained in $H_{F_{1}}^{2}\left(\mathbf{N}^{*}, Z\right)$.

In order to show equality we need some more computations. We must show that every cocycle represents the same class in $H_{F_{1}}^{2}\left(\mathbf{N}^{*}, Z\right)$ as a certain product of $f_{p, q}$ 's.

By the proof of 2.3 we know that the cohomology class of $f$ is equivalent to the class of $f^{\prime}$ where $f^{\prime}$ vanishes on all pairs $(a, 1)$ and $(1, a)$.

We start from subtracting from $f$ the product of elements $f(p, q) \cdot f_{p, q}$, where $p, q$ are prime numbers. This way we get a cocycle which vanishes at all pairs $(p, q)$ of prime numbers and all pairs $(a, 1),(1, a)$. We show that such $f$ is a coboundary in our cochain complex. This will be achieved by induction on the number of prime factors in the product of coordinates. Assume that our cocycle $f$ vanishes on all pairs $(v, w)$ such that $v w$ is a multiplication of at most $k$ primes. Assume that $x y$ is a product of $k+1$ primes and $f(x, y)=d$. We will modify $f$ inside its cohomology class to get a new cocycle which vanishes also at $(x, y)$.

Let $g: \mathbf{N}^{*} \rightarrow Z$ be defined by the formula $g(x y)=d$ and zero otherwise. Then $\left(f+\delta^{1} g\right)(x, y)=0$ as required. But of course $\delta^{1} g(s, t)=-d$ at all pairs $(s, t)$ for which $s t=x y$ and neither of $s$ and $t$ is 1 (and is 0 at all other points). We have to check that $f(s, t)=d$ at all such points. To see this observe that for any triple of different from 1 points $a, b$ and $c$ satisfying $a b c=x y=s t$ we have

$$
0=\delta^{2} f(a, b, c)=a f(b, c)-f(a b, c)+f(a, b c)-f(a, b) c
$$

because $f$ is a cocycle. By our assumption, that $f$ vanishes on all pairs $(v, w)$ for which $v w$ is a product of at most $k$-primes and by the assumption, that $x y$ is a product of precisely $k+1$ prime numbers we see that

$$
f(a, b c)=f(a b, c)
$$

Let $x=p_{1} p_{2} \ldots p_{i}$ and $y=p_{i+1} \ldots p_{k+1}$ where each $p_{j}$ is a prime number. Assume that $p_{1} \mid s$. Then by the formula above applied several times we get

$$
f(x, y)=f\left(p_{1}, p_{2} \ldots p_{k+1}\right)=f(s, t)
$$

If none of the $p_{j}$ s divides $s$ for $j=1, \ldots, i$ let $p_{m} \mid s$ for a certain $m \in\{i+1, \ldots, k+1\}$. Then

$$
\begin{gathered}
f(x, y)=f\left(p_{1}, p_{2} \ldots p_{k+1}\right)=f\left(p_{1} p_{m}, p_{2} \ldots p_{m-1} p_{m+1} \ldots p_{k+1}\right)= \\
=f\left(p_{m}, p_{1} p_{2} \ldots p_{m-1} p_{m+1} \ldots p_{k+1}\right)=f(s, t)
\end{gathered}
$$


This obviously implies the statement that $f(s, t)=d$ at all pairs satisfying $s t=x y$. So by induction on $k$ we know that our group $H_{F_{1}}^{2}\left(\mathbf{N}^{*}, Z\right)$ is generated by elements $f_{p, q}$. Let now $p$ and $q$ be two prime numbers and $g: \mathbf{N}^{*} \rightarrow Z$ be a function defined by the rule $g(p q)=-1$ and is 0 otherwise. Then $f_{p, q}+f_{q, p}-\delta^{1} g$ is a cocycle which vanishes at all pairs $(s, t)$ with prime $s$ and $t$. By our previous considerations we can say that it represents the trivial element in $H_{F_{1}}^{2}\left(\mathbf{N}^{*}, Z\right)$. Hence $\left[f_{p, q}\right]=\left[f_{q, p}\right]$ in $H_{F_{1}}^{2}\left(\mathbf{N}^{*}, Z\right)$. Similarly $f_{p, p}-\delta^{1} h$ vanishes at all pairs $(s, t)$ with prime $s$ and $t$, where $h\left(p^{2}\right)=-1$ and is zero otherwise. This observation finishes the proof of Theorem 2.4.

We finish the present section with classical considerations. The groups $H^{2}$ typically classify extensions in considered theories. Similarly we have here:

2.6. Theorem: The elements of the group $H_{F_{1}}^{2}\left(\mathbf{N}^{*}, Z\right)$ are in $1-1$ correspondence with isomorphism classes of monoids which are extensions of the multiplicative monoid $\mathbf{N}^{*}$ by the abelian group $Z$, where a monoid acts on a group by standard multiplication in $Z$.

Proof. Standard checking. Of course the result is the same as if we classify the extensions of the monoid ring $Z\left[\mathbf{N}^{*}\right]$ by a $Z\left[\mathbf{N}^{*}\right]$-module $Z$ in the category of $Z$-algebras.

\section{ABSOLUTE HOCHSCHILD COHOMOLOGY OF N*}

Let $\mathbf{W}_{\mathbf{n}}$ denote the free abelian monoid generated by $n$ elements $\left\{a_{1}, \ldots, a_{n}\right\}$. We will identify $\mathbf{W}_{\mathbf{n}}$ with the multiplicative submonoid of $\mathbf{N}^{*}$ generated by the first $n$ prime numbers. With this interpretation in mind we can view $\mathbf{N}^{*}$ as the direct limit of the $\mathbf{W}_{\mathbf{n}}$ 's. Observe that $Z\left[\mathbf{W}_{\mathbf{n}}\right]$ is isomorphic to the ring of polynomials over $Z$ with $n$ indeterminates. Hence its Hochschild cohomology groups are well known. Let us recall (see for example [W, Ex. 9.1.3]):

3.1. Lemma: Hochschild homology and cohomology groups of $Z\left[\mathbf{W}_{\mathbf{n}}\right]$ with any coefficients vanish above dimension $n$.

We will start our considerations with the simple lemma:

3.2. Lemma: Let $p$ be a prime number. Let $Z(p)$ denote the $\mathbf{W}_{\mathbf{1}}$ bimodule which is isomorphic to $Z$ as an abelian group and on which $a_{1}$ acts from both sides via multiplication by the prime number $p$. Let $M$ be any trivial $\mathbf{W}_{\mathbf{1}}$ bimodule which is free as an abelian group. Then

$$
\operatorname{Hoch}^{0}\left(Z\left[\mathbf{W}_{\mathbf{1}}\right], Z(p) \otimes M\right)=\operatorname{Hoch}^{1}\left(Z\left[\mathbf{W}_{\mathbf{1}}\right], Z(p) \otimes M\right)=Z \otimes M
$$

and

$$
\operatorname{Hoch}_{0}\left(Z\left[\mathbf{W}_{\mathbf{1}}\right], Z(p) \otimes M\right)=\operatorname{Hoch}_{1}\left(Z\left[\mathbf{W}_{\mathbf{1}}\right], Z(p) \otimes M\right)=Z \otimes M
$$

where tensor product means tensoring over $Z$. 
Proof. Calculation for $\operatorname{Hoch}^{0}$ and $H_{o c h}$ is obvious. We know that $\operatorname{Hoch}^{1}\left(Z\left[\mathbf{W}_{\mathbf{1}}\right], Z(p)\right)$ is equal to the group of derivations with values in $Z(p)$. So we have to calculate all the maps $\mathbf{W}_{\mathbf{1}} \rightarrow Z(p) \otimes M$ which satisfy the Leibniz rule. Let $f$ be a derivation and $f\left(a_{1}\right)=x$. It is obvious that $f(1)=0$ and from the Leibniz rule we get by induction that $f\left(a_{1}^{n}\right)=n p^{n-1} x$. Hence $\operatorname{Hoch}^{1}\left(Z\left[\mathbf{W}_{\mathbf{1}}\right], Z(p)\right)=Z \otimes M$ and, if we forget about $M$, it is generated by the derivation $g_{p}$ which sends $a_{1}$ to 1 . For the homological calculations observe first that $Z(p)$ is isomorphic to its dual also as $Z\left[\mathbf{W}_{\mathbf{1}}\right]$-bimodule. Hence we can use the ordinary universal coefficients formula relating Hochschild homology and cohomology:

$\left.\operatorname{Hoch}^{k}\left(Z\left[\mathbf{W}_{\mathbf{1}}\right], Z(p)\right)=\operatorname{Hom}_{(H o c h}\left(Z\left[\mathbf{W}_{\mathbf{1}}\right], Z(p)\right) ; Z\right) \oplus \operatorname{Ext}^{1}\left(\operatorname{Hoch}_{k-1}\left(Z\left[\mathbf{W}_{\mathbf{1}}\right], Z(p) ; Z\right)\right.$

From this we get our calculation for $M=Z$. But this implies the general case by our assumption on $M$.

Let $Q_{\left(p_{1}, \ldots, p_{n}\right)}^{*}$ denote the subgroup of the multiplicative group of positive rational numbers which consists of all fractions which (in not reducible form) are built out of the prime numbers $p_{1}, \ldots, p_{n}$ only. Then the map $\mathbf{W}_{\mathbf{n}} \rightarrow Q_{\left(p_{1}, \ldots, p_{n}\right)}^{*}$ which sends $a_{i}$ to $p_{i}$ embeds the monoid $\mathbf{W}_{\mathbf{n}}$ into a group $Q_{\left(p_{1}, \ldots, p_{n}\right)}^{*}$, the images of $a_{i}$ 's generate $Q_{\left(p_{1}, \ldots, p_{n}\right)}^{*}$ and every element of $Q_{\left(p_{1}, \ldots, p_{n}\right)}^{*}$ can be written as a fraction $x^{-1} y$ for some $x, y$ belonging to the image of $\mathbf{W}_{\mathbf{n}}$. So we are in the situation which is thoroughly studied in [CE, chapters 8 and 10]. We have:

3.3. Theorem: The embedding of multiplicative monoids $\mathbf{W}_{\mathbf{n}} \rightarrow Q_{\left(p_{1}, \ldots, p_{n}\right)}^{*}$ induces and isomorphism on Hochschild cohomology groups with $Q$ coefficients, where $Z\left[Q_{\left(p_{1}, \ldots, p_{n}\right)}^{*}\right]$ bimodule structure on $Q$ is given by multiplication of rational numbers from both sides.

Proof. The proof is essentially taken from [CE, chapter 8 and 10]. We will sketch it below for the readers' convenience because we are not able to quote one particular theorem from $[\mathrm{CE}]$ which implies immediately our theorem. Only for this proof let $A$ denote the monoid $\operatorname{ring} Z\left[\mathbf{W}_{\mathbf{n}}\right]$ and $B=Z\left[Q_{\left(p_{1}, \ldots, p_{n}\right)}^{*}\right]$. Let, as usual, $A^{e}$ and $B^{e}$ denote enveloping algebras of $Z$-algebras. Using the Ext-interpretation of Hochschild cohomology groups we have to show only that the natural embedding of algebras $A \mapsto B$ induces an isomorphism

$$
\operatorname{Ext}_{B^{e}}(B, Q) \rightarrow \operatorname{Ext}_{A^{e}}(A, Q)
$$

For this we will use Theorem 3.1 from [CE, chapter 8] where the necessary and sufficient condition is formulated for the map of augmented algebras to induce an isomorphism on Ext-groups with any coefficients. We have to check two conditions:

3.3.1. $\quad B^{e} \otimes_{A^{e}} A \simeq B$

3.3.2. $\operatorname{Tor}_{n}^{A^{e}}\left(B^{e}, A\right)=0$ for $n>0$

The first is easy: the natural map from the left to the right is certainly an epimorphism because the basic vector $1 \cdot p / q$ can be obtained as an image of $1 \otimes 1 / q \otimes p$. Assume now 
that $x$ goes to 0 . By finding common divisors and moving elements over the tensor product we can present $x$ as

$$
\sum_{i=1}^{k}\left(1 / b \otimes 1 / b \otimes p_{i}\right)
$$

where $p_{i}$ 's are arbitrary elements of $A$. The fact that $x$ maps to 0 in $B$ means that $\sum_{i=1}^{k} p_{i} / b^{2}=0$. This means that $\sum_{i=1}^{k} p_{i}=0$ which immediately implies that $x=0$.

For the second condition it is enough to show that

3.3.3 $B^{e}$ is a direct limit of projective $A^{e}$-modules.

Observe that our monoids are commutative so both $A^{e}$ and $B^{e}$ are monoid algebras over $Z$ of cartesian products of corresponding monoids. But in such a case 3.3.3 is fully shown as the second part of the proof of proposition 4.1 of chapter 10 in [CE].

3.4. Proposition: For any natural $k$ the group $\operatorname{Hoch}^{k}\left(Z\left[Q_{\left(p_{1}, \ldots, p_{n}\right)}^{*}\right], Q\right)$ is equal to the direct product of $Q$ indexed by all $k$-tuples of distinct prime numbers $q_{1}<q_{2}<\ldots<q_{k}$ taken from the set $\left\{p_{1}, \ldots, p_{n}\right\}$.

Proof. The monoid $Q_{\left(p_{1}, \ldots, p_{n}\right)}^{*}$ is an abelian group and hence its Hochschild cohomology groups are the same as the group homology with conjugation action on $Q$. But conjugation action is trivial so the groups of interest are equal to the cohomology of the group $Q_{\left(p_{1}, \ldots, p_{n}\right)}^{*}$ with trivial coefficients $Q$. Observe that $Q_{\left(p_{1}, \ldots, p_{n}\right)}^{*}$ is a free finitely generated abelian group generated by the numbers $p_{1}, p_{2}, \ldots, p_{n}$. Then our proposition is proved by the formula for the cohomology of a free finitely generated abelian group.

3.5. Proposition: The group $\operatorname{Hoch}^{k}\left(Z\left[\mathbf{W}_{\mathbf{n}}\right], Z\right)$ is equal to the direct product of $Z$ indexed by all $k$-tuples of distinct prime numbers $q_{1}<q_{2}<\ldots<q_{k}$ taken from the set $\left\{p_{1}, \ldots, p_{n}\right\}$.

Proof. This is an easy exercise from homological algebra and we will present only the way of reasoning leaving the details to the reader. The previous proposition gives us the size of the torsion free part of the groups of interest. Now use the cohomological Kunneth spectral sequence for the monoid $\mathbf{W}_{\mathbf{n}}=\mathbf{W}_{\mathbf{1}} \times \mathbf{W}_{\mathbf{n}-\mathbf{1}}$. By lemma 3.2 we know that the first table of this spectral sequence consists of two columns 0 and 1 (besides the trivial ones), each of which being equal to $C^{*}\left(Z\left[\mathbf{W}_{\mathbf{n}-\mathbf{1}}\right], Z\right)$. Hence the second table consists of two columns each of which filled with $\operatorname{Hoch}^{*}\left(Z\left[\mathbf{W}_{\mathbf{n}-\mathbf{1}}\right], Z\right)$. So they contain no torsion by induction on $n$ and by proposition 3.4 ("rank counting") we know that all differentials must be zero. From this our result follows immediately.

3.6. Theorem: The group $\operatorname{Hoch}^{k}\left(Z\left[\mathbf{N}^{*}\right], Z\right)$ is equal to the direct product of $Z$ indexed by all $k$-tuples of distinct prime numbers $q_{1}<q_{2}<\ldots<q_{k}$.

Proof. The proof is again based on the basic homological algebra methods. The monoid $\mathbf{N}^{*}$ is a direct limit of $\mathbf{W}_{\mathbf{n}}$ 's so from 3.5 we know that $\operatorname{Hoch}^{k}\left(Z\left[\mathbf{N}^{*}\right], Z\right)$ contains a subgroup isomorphic to the one described in the statement of the theorem coming from the inverse limit of the groups $\operatorname{Hoch}^{k}\left(Z\left[\mathbf{W}_{\mathbf{n}}\right], Z\right)$. To show that this is all of it we need 
some more arguments. Observe that we can perform the same calculations for Hochschild homology of $\mathbf{W}_{\mathbf{n}}$ as were done for cohomology in 3.5. We get then that $H \operatorname{Hoch}_{k}\left(Z\left[\mathbf{W}_{\mathbf{n}}\right], Z\right)$ are, as abelian groups, the same as cohomology. But homology of monoids commute with direct limits hence we calculate that $\operatorname{Hoch}_{k}\left(Z\left[\mathbf{N}^{*}\right], \mathbf{Z}\right)$ is a direct sum of $Z$ indexed by all $k$-tuples of distinct prime numbers. Now observe (compare the proof of 3.2) that $Z$ is isomorphic to its dual as bimodules over $Z\left[\mathbf{N}^{*}\right]$ and hence we get our result for cohomology by the universal coefficients formula.

3.7. Remark: We know now the group $H_{F_{1}}^{n}\left(\mathbf{N}^{*}, Z\right)$ for any natural $n$. It is equal to the infinite product of $Z$ indexed by all $n$-tuples of prime numbers $\left(p_{1}<p_{2}<\ldots<p_{n}\right)$. We leave it to the interested reader to check directly from the definitions that the classes $f_{\left(p_{1}, \ldots, p_{n}\right)}$ form a basis of it (in the sense of an infinite product).

\section{RELATION TO TOPOLOGICAL HOCHSCHILD COHOMOLOGY.}

In this section we will relate absolute mathematics to better known objects. In [KOW] and $[\mathrm{O}]$ the problem of finding $H_{F_{1}}^{1}$ for matrix rings $M_{n}(R)$ was studied for different $R$ and $n$. In our language this is the same as to try to calculate Hochschild cohomology

$$
\operatorname{Hoch}^{1}\left(Z\left[\mathbf{M}_{\mathbf{n}}(\mathbf{R})\right], M_{n}(R)\right)
$$

with bimodule structure defined by multiplication of matrices from both sides. This problem with growing $n$ was studied in [BP]. The expression "growing $n$ " means here that we study the map on (co)homology induced by the embedding of $M_{n}(R)$ into $M_{n+1}(R)$ and we are interested in the (co)limit of $\operatorname{Hoch}^{i}\left(Z\left[\mathbf{M}_{\mathbf{n}}(\mathbf{R})\right], M_{n}(R)\right)$ when $n$ tends to infinity (actually the more complicated coefficients where considered in [BP]) . Theorem 1.3 of [BP] says that the map described above is an isomorphism provided $n>2^{i+1}$. It means that for $i=1$ stability starts at $n=5$. On the other hand, by [JP] (see also [B, lemma 3.2]) the stable groups are isomorphic to $H_{M} L^{*}(R, R)$ - the MacLane cohomology groups of the ring $R$ with coefficients in the bimodule $R$. So absolute Hochschild (co)homology theory can be viewed as unstable version of MacLane theory or we can interpret the latter one as stabilization of the former. The MacLane groups for $R=Z$ were calculated in [FP]. In particular it was shown there that $H M L^{1}(Z, Z)=0$. Hence as an immediate corollary we get the following result about absolute mathematics:

\subsection{Corollary: $H_{F_{1}}^{1}\left(\mathbf{M}_{\mathbf{n}}(\mathbf{Z}), M_{n}(Z)\right)=0$ for $n>4$.}

Of course in the corollary above we can replace $Z$ by any ring for which $H M L^{1}(R, R)=$ 0 . In $[\mathrm{O}]$ the result was shown for $n>2$ and rings $R$ contained in the algebraic closure of $Q$. For $n>4$ we can extend this result to other rings. We have the following calculation:

4.2.Theorem: Let $k=Z$ or $k=F_{p}$. Let $R$ be an algebra over $k$ which is projective as a $k$-module. Then

$$
H_{F_{1}}^{1}\left(\mathbf{M}_{\mathbf{n}}(\mathbf{R}), M_{n}(R)\right)=\operatorname{Hoch}^{1}(R, R)
$$

for $n>4$. 
Proof. We have described at the beginning of this section the relation between absolute Hochschild theory and MacLane cohomology. After that it is obvious that we have only to show that $H M L^{1}(R, R)=\operatorname{Hoch}^{1}(R, R)$. We will follow the lines of $[\mathrm{P}]$. Let us recall, that any $R$-bimodule $M$ can be used as coefficients in MacLane cohomology groups. Using precisely the same method as was used in [P, Proposition 4.1] one can construct a cohomological type spectral sequence for any $R$-bimodule $M$

$$
E_{2}^{p, q}=\operatorname{Hoch}^{p}\left(R, H M L^{q}(k, M)\right) \Rightarrow H M L^{p+q}(R, M)
$$

(The only difference here comparing to $[\mathrm{P}]$ is to start from the resolution of the identity functor and not its dual.) For our purposes we put $M=R$. In both cases, which we consider here, $H M L^{1}(k, k)=0$ and hence $H M L^{1}(k, R)=0$ by our assumption on $R$. On the other hand $H M L^{0}(k, R)=R$ because $k$ commutes with the elements of $R$ and the only matrix over $R$ which commutes with all matrices over $k$ is the diagonal one. Hence we get our calculation and the proof is finished.

4.3.Remark: Theorem 4.2 shows that for $Z$ or $F_{p}$ algebra $R$ vanishing of absolute Hochschild groups of large matrices over $R$ is implied by the vanishing of $\operatorname{Hoch}^{1}(R, R)$.

4.4.Remark: It is clear that the method used in the proof of 4.2 can be extended to algebras over other ground rings. We wanted here to present the method of attacking absolute problems rather than the most general results in this direction. The interesting feature, which should have some explanation, lies in the fact that absolute mathematics of the ring $R$ seems to be simpler when $R$ is less commutative (rings versus matrices over them).

4.5.Problem: As we have said before MacLane cohomology groups of the ring $Z$ were calculated in $[\mathrm{FP}]$. They are torsion and given by the formula $H M L^{2 i-1}(Z, Z)=Z / i$ and vanish in other dimensions. As we have shown in sections 2 and 3 the absolute Hochschild cohomology groups of $Z$ are huge but contain only 2-torsion. Do the 2-torsion classes from $H M L^{*}(Z, Z)$ map nontrivially by the stability homomorphisms to the absolute Hochschild cohomology of $Z$ ? In other words, do the 2-torsion classes in $H M L^{*}(Z, Z)$ describe phenomena related to the multiplication of integers or, perhaps, they describe only phenomena which are related to larger and larger matrices.

Acknowledgment: This research was partially supported by the Polish Scientific Grant 1 P03A 00526. 


\section{References:}

[B] S. Betley. On stable K-theory and topological Hochschild homology. Comm. Math. Helv. 67 (1992) 394-405.

[BP] S. Betley, T. Pirashvili. Twisted (co)homological stability for monoids of endomorphisms. Math. Ann. 295 (1993) 709-720.

[CE] H. Cartan, S. Eilenberg. Homological algebra. Princeton University Press 1956, Princeton, New Jersey.

[FP] V. Franjou, T. Pirashvili. On MacLane cohomology of the ring of integers. Topology 37 (1998) 109-114.

[JP] M. Jibladze, T. Pirashvili. Cohomology of algebraic theories. J. Algebra 137 (1991) 253-296.

[KOW] N. Kurokawa, H. Ochiai, M. Wakayama. Absolute derivations and zeta functions. Doc. Math. , Extra Vol. Kato, (2003) 565-584.

[O] N. Kurokawa, H. Ochiai. On monoidal absolute derivations for non-commutative rings. J. Ramanujan Math. Soc. 19 (2004) 261-265.

[P] T. Pirashvili. Spectral sequence for Mac Lane Homology. J. Algebra 170 (1994) 422-428.

[S] C. Soule. On the field with one element. Max-Planck-Institute fur Mathematik, Arbeitstagung 1999 , 5 pages; IHES prepublications M/55,1999.

[W] C. Weibel. An introduction to homological algebra. Cambridge Studies in Advanced Mathematics 38, Cambridge University Press 1994.

\section{Instytut Matematyki, University of Warsaw ul.Banacha 2, 02-097 Warsaw, Poland e-mail: betley@mimuw.edu.pl}

